\title{
Financial feasibility analysis in the implementation of a photovoltaic microgeneration system
}

\author{
Filipe Alves Barboza ${ }^{1}$, Lucas Lira Souza ${ }^{2}$, Adeon Cecilio Pinto ${ }^{3}$
}

1,2,3 Universidade Federal do Vale do São Francisco (UNIVASF). Av. José de Sá Maniçoba, S/N - Centro, Petrolina - PE.

Email: felipibarbosa96@gmail.com, lucaslira.nls@gmail.com, adeon.pinto@univasf.edu.br

Received: December $21^{\text {th }}, 2018$

Accepted: January $15^{\text {th }}, 2019$

Published: March $31^{\text {th }}, 2019$

Copyright (02016 by authors and Institute of Technology Galileo of Amazon (ITEGAM).

This work is licensed under the Creative Commons Attribution International

License (CC BY 4.0).

http://creativecommons.org/licenses/by/4.0/

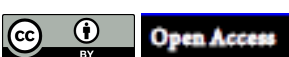

\begin{abstract}
This article presents an economic feasibility evaluation for a possible installation of a photovoltaic system in a family home in the state of Pernambuco, which has high consumption. In order to reach the objective outlined, a survey of costs related to the equipment and energy tariffs practiced by the Electric Company of Pernambuco (CELPE) was carried out in the last 17 years, aiming to transmit veracity to the study in question. In the financial analysis, the main tools used in project evaluations were used, such as Net Present Value (NPV), Payback, and Internal Rate of Return (IRR). These indicators provide information that helps decision making and bring to light the liquidity capacity of the application, as well as its profitability. After applying these techniques, it was possible to observe that the investment returns the invested capital in a few years, when compared to its useful life, and presents a high degree of profitability, being its income superior up to three times of the initial value. Thus, it is safe to say that, under the conditions worked, the viability of the project is evident. Thus, in view of financial attractiveness, we sought to encourage and disseminate this type of energy generation through an economic approach.
\end{abstract}

Keywords: Economic feasibility, financial analysis, investments, photovoltaic system.

\section{Análise de viabilidade financeira na implantação de um sistema de microgeração fotovoltaica}

\section{RESUMO}

Este artigo traz uma avaliação de viabilidade econômica para uma possível instalação de um sistema fotovoltaico em uma residência familiar, no estado de Pernambuco, que possui alto consumo. Para atingir o objetivo traçado, foi realizado um levantamento de custos relativos ao equipamento, e às tarifas de energia praticadas pela Companhia Elétrica de Pernambuco (CELPE) nos últimos 17 anos, buscando transmitir veracidade ao estudo em questão. Na análise financeira, utilizou-se as principais ferramentas empregadas em avaliações de projetos, sendo elas o Valor Presente Líquido (VPL), o Payback, e a Taxa Interna de Retorno (TIR). Tais indicadores fornecem informações que auxiliam o processo decisório e trazem à luz a capacidade de liquidez da aplicação, assim como sua rentabilidade. Após a aplicação dessas técnicas, foi possível observar que o investimento retorna o capital investido em poucos anos, quando comparado à sua vida útil, e apresenta alto grau de lucratividade, sendo sua renda superior em até três vezes do valor inicial. Sendo assim, é seguro afirmar que, nas condições trabalhadas, a viabilidade do projeto é evidente. Dessa forma, tendo em vista a atratividade financeira, buscou-se incentivar e disseminar esse tipo de geração de energia, através de uma abordagem econômica.

Palavras-Chave: Viabilidade econômica, análise financeira, investimentos, sistema fotovoltaico.

\section{INTRODUÇÃO}

A ideia de utilização de fontes alternativas de energias adquiriu grande notoriedade nos últimos anos devido, principalmente, a conscientização da provável escassez dos recursos fósseis, por exemplo petróleo, e a necessidade de reduzir as emissões de gases nocivos à atmosfera. Um exemplo das fontes de energia são os painéis fotovoltaicos que 
transformam a energia proveniente dos raios solares incidentes em energia elétrica.

O Brasil conta com um enorme potencial para geração de energia solar devido a sua localização geográfica, onde a maior parte de seu território situa-se próximo à linha do equador, consequentemente recebe a incidência de luz praticamente constante durante todo o dia. Todavia, esta ainda segue negligenciada pela falta de incentivo financeiro e de políticas que estimulem a utilização de novos meios para produção de energia.

O não/mau aproveitamento de recursos alternativos causa uma dependência de outras fontes. As hidrelétricas dominam a matriz de energia brasileira, como é possível observar na Figura 1, seguidas por fontes não renováveis, sendo estas uma segunda via para geração.

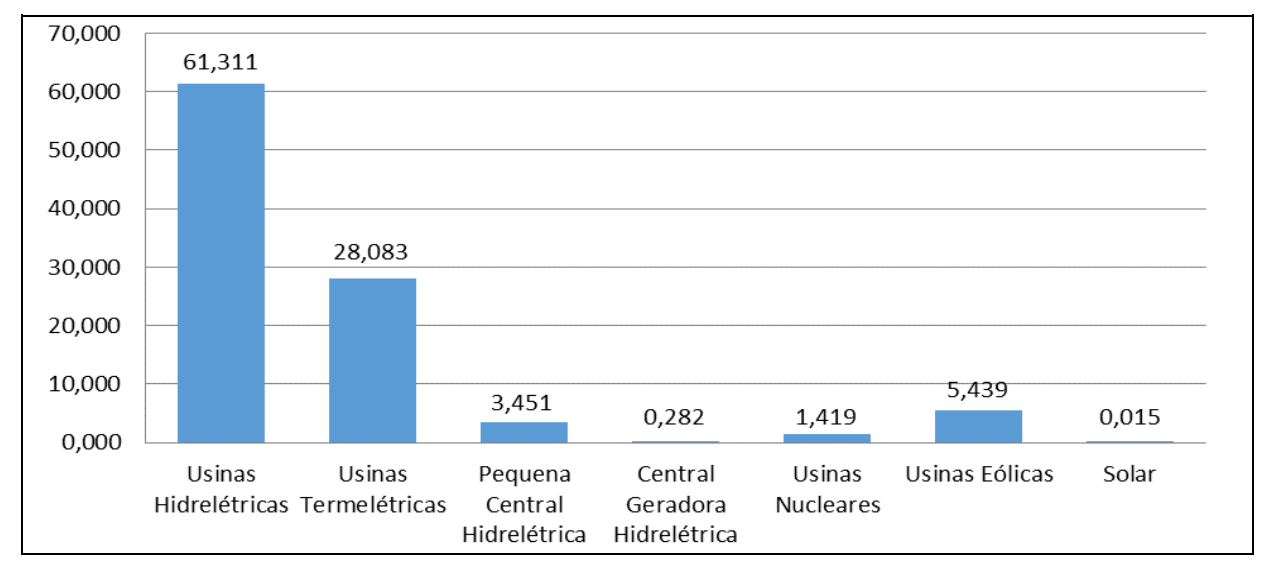

Figura 1: Capacidade Instalada de Geração Elétrica no Brasil (em porcentagem) Fonte: Adaptado de [1].

A fonte hidráulica, apesar de ser renovável acarreta uma série de impactos ambientais e exige habilidade gerencial para administrar esses enormes empreendimentos. Ademais, devido aos níveis de pluviosidade estarem abaixo dos normais em áreas específicas, a utilização de usinas termelétricas como meio de suprir a energia demandada, tornou-se uma atividade frequente. Estas possuem alto custo quando ativadas, resultando em aumentos no sistema tarifário, e influenciando, consequentemente, no orçamento população, que paga pelo custo extra.

O sistema tarifário brasileiro é, segundo [2], a estruturado em um conjunto de tarifas, as quais variam de acordo com o grupo o qual estão sendo aplicadas. A tarifa corresponde ao valor monetário da energia elétrica $(\mathrm{R} \$ / \mathrm{kWh})$, e para as residências, que se encontram no subgrupo B1 [3], ela é aplicada de forma convencional, ou seja, caracteriza-se por um valor fixo que independe do horário de utilização do dia ou época do ano.

Como expresso anteriormente, a capacidade para geração de energia solar é vasta, tendo em vista as particularidades territoriais. Esta pode ser utilizada como segunda opção para produção de energia em ocasiões na qual a geração convencional através das hidrelétricas não seja suficiente, tornando assim desnecessário o uso das termelétricas.

De acordo com o [4] a instalação de sistemas fotovoltaicos tem se tornada atrativa nos últimos anos devido à redução de custo contínua dos painéis. O preço dos mesmos é agora cerca da metade do preço que eles estavam em 2008, e cerca de 100 vezes menor do que eram em 1977. Ademais, esse sistema possui uma vida útil de aproximadamente 25 anos, não necessitando de manutenções periódicas frequentes, mostrando-se um investimento a longo prazo, o qual têm retorno mensal durante seu ciclo de vida, diminuindo seus custos à medida do uso.

As condições para o desenvolvimento dessa forma de produção energética estão cada vez mais favoráveis, visto que apresenta uma trajetória de custos decrescentes ano após ano $[5]$.

Segundo o [6], os painéis fotovoltaicos, geralmente fabricados de Silício cristalino, são $99 \%$ recicláveis e produzem energia elétrica por mais de 40 anos sem poluir. A grande maioria desses chegam ao final de vida útil com $80 \%$ de sua potência original. A eficiência comercial varia de $13 \%$ a $16 \%$, sendo os que possuem um índice superior a $16 \%$ considerados premium, os quais evidentemente, apresentam maiores custos.

A Agência Nacional de energia Elétrica (ANEEL) aprovou a Resolução Normativa 687/2015, a qual faz modificações na Resolução 482/2012, classificando os sistemas de micro e minigeração distribuída, buscando reduzir as barreiras econômicas e facilitar a inserção dessa fonte alternativa, através do sistema de compensação de energia [1].

O sistema de compensação baseia-se em créditos de energia, quando a energia gerada com a energia consumida apresentarem um balanço líquido positivo, são gerados créditos que podem ser utilizados para abater o consumo nos meses subsequentes por um período de até 60 meses. Portanto, é possível que o consumidor de energia elétrica passe a gerar energia para consumo próprio e exportação dos excedentes para a rede elétrica [5].

No que refere-se à literatura acerca do tema que será abordado, é observado que esta ainda permanece escassa e o conteúdo científico apresentado por demais autores apresentam limitações. É constatado que para realização de estudo de viabilidade econômica para investimentos de longo prazo, fazse necessário calcular a variação dos valores que estiverem em função das taxas flutuantes do mercado, porém devido à dificuldade de realizar tal cálculo, alguns autores optam por manter estas taxas fixas ao longo do tempo de estudo [7-8].

Todavia, a pesquisa em destaque realizará uma projeção para mensurar a variação dessas taxas, utilizando de valores anteriores à data da atual pesquisa e estimando as futuras alterações que acontecerão ao longo do tempo de investimento 
estipulado. A utilização desse artifício tem como objetivo atenuar as discrepâncias entre os valores teóricos que serão apresentados e os valores reais.

Apesar de toda atratividade e de fatores positivos, socioeconômicos e ambientais, a geração de energia solar ainda não está difundida significativamente. Diante disso, o presente artigo tem como objetivo, através da simulação de uma implantação de um sistema fotovoltaico, realizar uma análise de viabilidade econômica, empregando técnicas de análise de investimento. O estudo terá como base uma residência na qual o consumo médio é de $570 \mathrm{kWh}$, no estado de Pernambuco. Dado tal enfoque, buscar-se-á destacar aspectos favoráveis à utilização da microgeração, evidenciando indicadores financeiros que auxiliem no processo decisório do projeto. Procurando informar e trazer tal tecnologia à realidade, fez-se necessária uma pesquisa dessa natureza, a fim de avaliar aspectos relativos à utilização da energia solar fotovoltaica.

\section{METODOLOGIA}

A pesquisa é configurada pela realização de um estudo de viabilidade econômica, fundamentada no levantamento de dados referentes aos custos de um sistema fotovoltaico, avaliando sua rentabilidade e liquidez.

$\mathrm{O}$ estudo trata-se de caráter científico acadêmico, tendo por método a pesquisa quantitativa, buscando interpretar resultados obtidos numericamente, a fim de trazer insights que possibilitem uma tomada de decisão correta acerca da situação.

Este artigo caracteriza-se no método exploratório, utilizando-se da investigação de fatos e fazendo uma análise do que se sucede na realidade, na qual os dados são interpretados com respaldo em um embasamento teórico consistente, tencionando explorar a problemática estabelecida.

Tem como foco proporcionar maior familiaridade com o problema, de forma a torná-lo mais explícito. Pode-se dizer que esse tipo de pesquisa têm como objetivo principal o aprimoramento de ideias ou a descoberta de intuições [9].

A estrutura da análise financeira consiste em 3 etapas: estimativa de custos; definição de taxas; e aplicação de métodos.

Inicialmente serão estimados todos os custos que envolvem o processo de implementação do sistema, sejam eles de materiais, de instalação ou de planejamento. Em seguida serão determinadas as informações necessárias para a execução das técnicas financeiras. E por fim, considerando os custos associados à primeira etapa, juntamente com os resultados da segunda, faz-se a aplicação dos indicadores.

\section{II.1 ANÁLISE DE INVESTIMENTOS}

A análise de investimentos estuda a aplicação de recursos, baseando-se em artifícios financeiros para apontar a melhor alocação de investimento, considerando os custos precedentes e resultados posteriores, a fim de proporcionar rentabilidade e identificar capacidade de liquidez e segurança em projetos.

Com o intuito de realizar uma pesquisa abrangente e prover resultados confiáveis, utilizar-se-á três métodos para avaliar a viabilidade na implantação de um sistema fotovoltaico. Isso decorre do fato de que cada procedimento possui limitações e que a aplicação de apenas um deles não é satisfatória para obter respostas consistentes.
De acordo com [10], o conhecimento desses métodos é importante para apoiar a boa qualidade da tomada de decisão de investimentos.

\section{II.2 TÉCNICAS DE ANÁLISE DE INVESTIMENTOS}

\section{II.2.1 VALOR PRESENTE LÍQUIDO (VPL)}

O Valor Presente Líquido (VPL) ou Net Present Value (NPV), é considerado um método sofisticado, o que o torna um dos mais empregados em análise financeira de projetos, sendo possível calcular o valor do dinheiro no tempo levando em consideração as taxas de juros correntes. Por meio deste, as entradas e saídas de recursos (cash-flow) projetadas são trazidas para o valor atual, possibilitando ser comparadas ao investimento.

Através do VPL, os fluxos de retorno do investimento são descontados a uma taxa específica. Esta taxa, chamada de taxa de desconto se refere ao retorno mínimo que um investimento deve ter para ser considerado atrativo, de forma a cobrir o seu custo de oportunidade [11].

O custo de oportunidade é a avaliação de quanto está custando perder a oportunidade de investir em outra coisa, que tem por característica ser líquida e certa [12].

A taxa de desconto, também conhecida como taxa média de atratividade (TMA), segundo [13], baseia-se em fatores como natureza do negócio, objetivo do investimento, custo dos fundos de garantia para o investimento e taxa de retorno mínimo desejável e é sempre estabelecida pela gerência.

Através do valor do VPL, define-se a viabilidade ou não do investimento. Se este for maior que zero, considera-se o projeto atrativo, já que possui rentabilidade e é capaz de remunerar o capital aplicado com garantia. Quando o VPL tiver valor inferior a zero, o investimento é classificado como inviável, pois seu rendimento não será capaz de cobrir integralmente os custos, ocasionando prejuízos. Se igual a zero, julga-se o investimento como indiferente, este não apresentará rentabilidade, porém não será capaz de gerar perdas.

O cálculo do valor presente líquido se dá por meio da equação abaixo.

$$
V P L=\sum_{n=0}^{N} \frac{F C_{n}}{(1+T M A)^{n}}
$$

O termo $\mathbb{N}$ representa o número de parcelas, $F C_{n}$ representa o fluxo de caixa no período $n$, sendo a primeira parcela contabilizada negativa, pois refere-se ao investimento inicial e a TMA (taxa média de atratividade) representa o mínimo valor de retorno que o investidor pretende obter.

A técnica VPL não consegue fornecer os benefícios e prejuízos de um investimento. Para estudar a viabilidade financeira deverão ser utilizados outros parâmetros complementares, como a Taxa Interna de Retorno (TIR).

\section{II.2.2 TAXA INTERNA DE RETORNO (TIR)}

A Taxa Interna de Retorno (TIR) ou Internal Rate of Return (IRR), consiste na taxa de desconto que iguala o VPL a zero, fazendo com que as entradas (inputs) se igualem as saídas 
(outputs), isto é, representa o lucro real a ser acumulado no investimento.

Para [14], realizar uma análise pela TIR é extrair do projeto o percentual de ganho que ele oferece ao investidor.

Empregando essa técnica, o critério a ser avaliado consiste na comparação entre a TIR e a TMA. Se a taxa interna de retorno for maior que a taxa média de atratividade traçada inicialmente, o investimento é tido como viável, caso contrário, é tomado como inviável.

De acordo com [11], a TIR possui como maior vantagem possibilitar a comparação de investimentos de diferentes tipos e de considerar o prazo total e a escala dos mesmos, pois ela tem o caráter relativo, expressando seus resultados percentualmente, e não em valores absolutos, como o VPL.

\section{II.2.3 PAYBACK TIME}

O termo payback significa "retorno" e representa um indicador utilizados pelas empresas para mensurar o período de tempo de retorno financeiro de um investimento, ou seja, o tempo necessário para igualar todos os rendimentos provenientes de um investimento ao o valor inicial deste.

As principais vantagens apresentadas pelo cálculo do payback são principalmente a sua fórmula simples e de fácil aplicação, oferecendo uma estimativa do nível de risco de um investimento. Em contrapartida, apresenta algumas desvantagens, pois para projetos de longa duração esses rendimentos podem apresentar uma grande taxa de variação, dificultando assim o cálculo.

O cálculo do payback envolve uma fórmula simples, pois todas as variáveis são fáceis de serem estimadas. Ele consiste numa divisão do investimento inicial pelo resultado da média de rendimentos provenientes desse investimento [15].

Todavia, a simplicidade do payback o torna um método incorreto na perspectiva matemática, pois não considera os princípios de equivalência de taxas ao longo do tempo estabelecidos pela matemática financeira. E para suprir essa deficiência seu cálculo foi aprimorado e denominado payback descontado, que por sua vez utiliza uma taxa de desconto que representa a valorização do capital ao longo do tempo. A taxa de desconto usualmente utilizada é a TMA (Taxa Mínima de Atratividade).

Para o novo cálculo é necessário descontar a taxa utilizada dos fluxos de caixas presentes no período de investimento analisado, somando os valores das referidas parcelas por fim realizar o cálculo do payback original.

\section{RESULTADOS E DISCUSSÕES}

Seguindo a divisão proposta na metodologia, foi feito um levantamento de dados através de uma pesquisa de mercado, buscando quantificar custos e descrevê-los, assim como todas as informações relativas à instalação de um sistema. A Tabela 1 mostra tais custos.

Foi escolhido, para esse estudo, uma residência onde o consumo médio mensal é de $570 \mathrm{kWh}$, na qual o equipamento fotovoltaico teria capacidade para gerar $584 \mathrm{kWh} / \mathrm{mês}$, de forma que seria compensado $108 \%$ do consumo da casa. Para atender essa demanda de energia, o sistema necessita de 16 painéis fotovoltaicos, fazendo-se necessário utilizar uma área de $25,60 \mathrm{~m}^{2}$ e tendo capacidade total de 4,32 $\mathrm{kWp}$.
Tabela 1: Custos do projeto.

\begin{tabular}{|c|c|}
\hline Descrição & Total \\
\hline Kit Gerador Solar Fotovoltaico & $\mathbf{R} \$ \mathbf{2 5 . 2 9 1 , 0 0}$ \\
\hline Custos de Instalação & $\mathbf{R} \mathbf{3 . 4 5 6 , 0 0}$ \\
\hline Layout de Projeto & $\mathbf{R} \mathbf{5 0 0 , 0 0}$ \\
\hline Total & $\mathbf{R} \$ \mathbf{2 9 . 2 4 7 , 0 0}$ \\
\hline Fonte: Autores, (2018)
\end{tabular}

No que tange à manutenção dos equipamentos, esta ocorre apenas esporadicamente, tendo um custo mínimo, quando comparada ao do sistema, e variável, visto que depende de qual empresa/estabelecimento irá realizá-la. Os custos de adaptação para instalar o mecanismo também são variáveis e não há ao certo como mensurá-los, pois baseiam-se na estrutura da residência.

Para poder aplicar o método VPL, é necessário calcular o retorno financeiro em pelo menos um ano, só a partir deste é possível projetar as próximas entradas de capital. Sabendo que o sistema gera $7008 \mathrm{kWh} /$ ano e o consumo é de $6840 \mathrm{kWh} / \mathrm{ano}$, considerando a tarifa de energia média de 2017, tem-se a seguinte situação descrita na Tabela 2.

Tabela 2: Consumo e geração.

\begin{tabular}{|c|c|c|}
\hline $\begin{array}{c}\text { Consumo } \\
\text { Anual (kWh) }\end{array}$ & $\begin{array}{c}\text { Tarifa } \\
(\mathbf{R S} / \mathbf{k W h})\end{array}$ & Despesa Anual \\
\hline 6840 & 0,66557 & $\mathrm{R} \$ 4.552,50$ \\
\hline $\begin{array}{c}\text { Geração } \\
\text { Anual (kWh) }\end{array}$ & $\begin{array}{c}\text { Tarifa } \\
(\mathbf{R} \$ \mathbf{k W h})\end{array}$ & Economia Anual \\
\hline 7008 & 0,66557 & $\mathrm{R} \$ 4.664,31$ \\
\hline
\end{tabular}

Fonte: Autores, (2018).

Feito esse cálculo, pode-se construir o fluxo financeiro. Entretanto, as entradas não serão as mesmas a cada ano, visto que a tarifa energética não se mantém constante, sofrendo variações ao longo do tempo. Dessa forma, procurando estabelecer um balanceamento, foi feita uma média da variação das tarifas entre os anos de 2001 e 2017, como é possível observar na tabela 3. Na figura 2, essa variação é posta de forma gráfica em valores percentuais para melhor compreensão. É importante ressaltar que as tarifas aqui trabalhadas já recebem incidência de impostos, e que estes estão sendo considerados nos cálculos realizados.

Tabela 3: Média das tarifas.

\begin{tabular}{|c|c|c|}
\hline Ano & Variação & \multirow{2}{*}{ Média } \\
\hline 2001 & & \\
\hline 2002 & $14,161 \%$ & \\
\hline 2003 & $21,528 \%$ & \\
\hline 2004 & $32,374 \%$ & \\
\hline 2005 & $19,231 \%$ & \\
\hline 2006 & $17,526 \%$ & \\
\hline 2007 & $3,492 \%$ & \\
\hline 2008 & $1,214 \%$ & \multirow{3}{*}{$8,9166 \%$} \\
\hline 2009 & $-0,940 \%$ & \\
\hline 2010 & $3,353 \%$ & \\
\hline 2011 & $-3,142 \%$ & \\
\hline 2012 & $3,642 \%$ & \\
\hline 2013 & $-15,182 \%$ & \\
\hline 2014 & $15,481 \%$ & \\
\hline 2015 & $4,761 \%$ & \\
\hline 2016 & $16,936 \%$ & \\
\hline 2017 & $8,230 \%$ & \\
\hline
\end{tabular}

Fonte: Autores, (2018). 


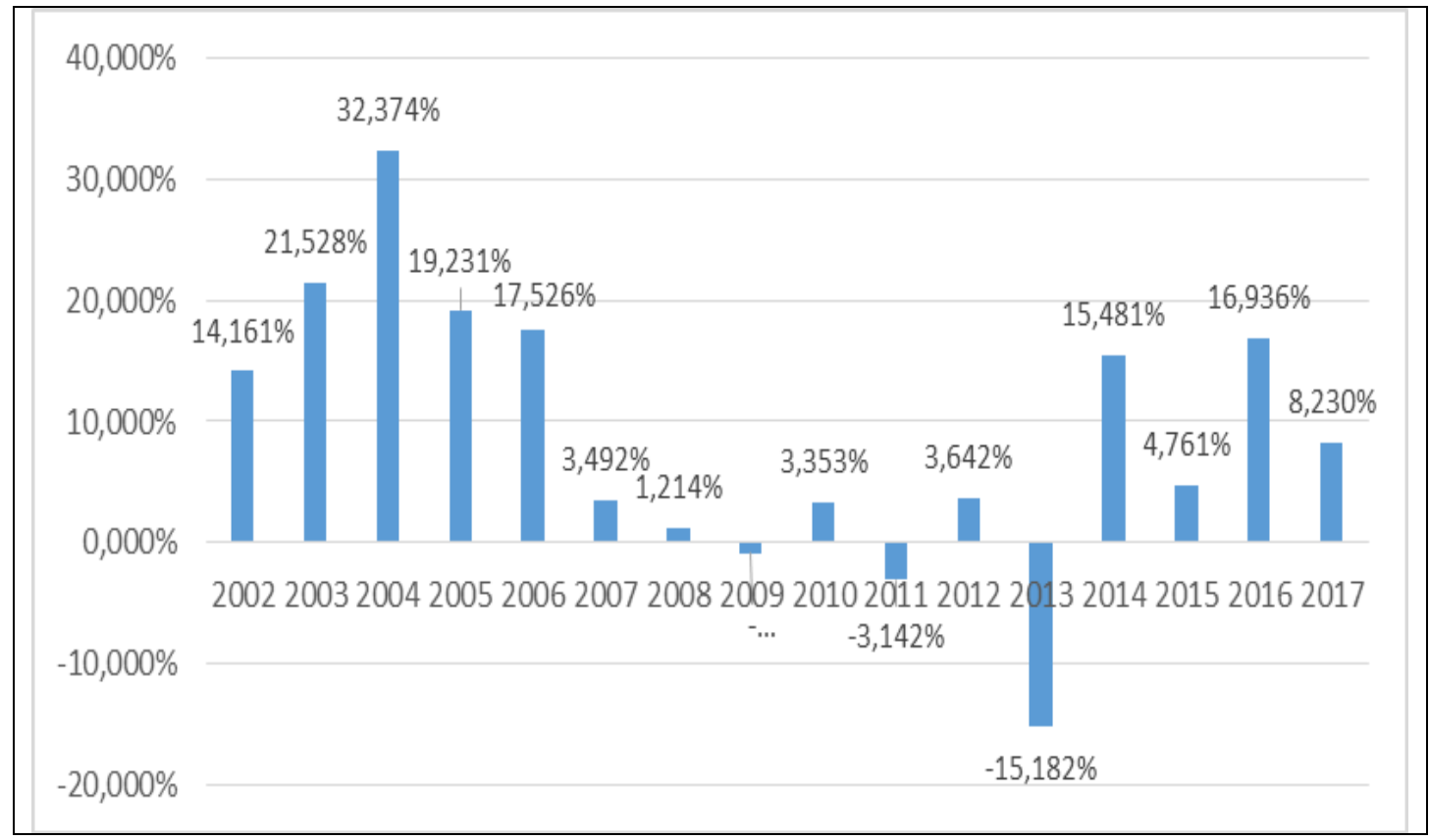

Figura 2: Variação tarifária percentual em relação ao ano anterior. Fonte: Autores, (2018).

Para de fato calcular o valor presente líquido, utiliza-se a taxa média de atratividade (TMA), a qual será considerada a taxa de rendimento anual da poupança, 6,7\% a.a. Esta foi determinada com base no retorno mínimo necessário para que o investimento em energia solar se mostrasse lucrativo. As projeções do fluxo de caixa do investimento foram realizadas a partir da primeira e sendo aplicada sobre esta a alteração média da tarifa de energia. Na tabela abaixo tem-se o fluxo de caixa e o VPL.

Tabela 4: Cash-flow e VPL.

\begin{tabular}{|c|c|c|c|}
\hline Período & Ano & \multicolumn{2}{|c|}{ Cash-flow } \\
\hline 0 & 2017 & $-\mathrm{R} \$$ & $29.247,00$ \\
\hline 1 & 2018 & $\mathrm{R} \$$ & $4.552,50$ \\
\hline 2 & 2019 & $\mathrm{R} \$$ & $4.958,43$ \\
\hline 3 & 2020 & $\mathrm{R} \$$ & $5.400,55$ \\
\hline 4 & 2021 & $\mathrm{R} \$$ & $5.882,10$ \\
\hline 5 & 2022 & $\mathrm{R} \$$ & $6.406,58$ \\
\hline 6 & 2023 & $\mathrm{R} \$$ & $6.977,83$ \\
\hline 7 & 2024 & $\mathrm{R} \$$ & $7.600,01$ \\
\hline 8 & 2025 & $\mathrm{R} \$$ & $8.277,67$ \\
\hline 9 & 2026 & $\mathrm{R} \$$ & $9.015,76$ \\
\hline 10 & 2027 & $\mathrm{R} \$$ & $9.819,66$ \\
\hline 11 & 2028 & $\mathrm{R} \$$ & $10.695,24$ \\
\hline 12 & 2029 & $\mathrm{R} \$$ & $11.648,89$ \\
\hline 13 & 2030 & $\mathrm{R} \$$ & $12.687,58$ \\
\hline 14 & 2031 & $\mathrm{R} \$$ & $13.818,88$ \\
\hline 15 & 2032 & $\mathrm{R} \$$ & $15.051,05$ \\
\hline 16 & 2033 & $\mathrm{R} \$$ & $16.393,10$ \\
\hline 17 & 2034 & $\mathrm{R} \$$ & $17.854,80$ \\
\hline 18 & 2035 & $\mathrm{R} \$$ & $19.446,84$ \\
\hline 19 & 2036 & $\mathrm{R} \$$ & $21.180,84$ \\
\hline 20 & 2037 & $\mathrm{R} \$$ & $23.069,45$ \\
\hline 21 & 2038 & $\mathrm{R} \$$ & $25.126,46$ \\
\hline 22 & 2039 & $\mathrm{R} \$$ & $27.366,89$ \\
\hline 23 & 2040 & $\mathrm{R} \$$ & $29.807,08$ \\
\hline 24 & 2041 & $\mathrm{R} \$$ & $32.464,86$ \\
\hline 25 & 2042 & $\mathrm{R} \$$ & $35.359,63$ \\
\hline VPL & \multicolumn{3}{|c|}{ R \$ 108.773,81 } \\
\hline
\end{tabular}

Fonte: Autores, (2018).
Tratando-se do payback, foi descontado no valor inicial do investimento, o valor da economia anual de energia, e assim sucessivamente com os valores atualizados mediante a taxa média de aumento, até 25 anos subsequentes. A partir desse mecanismo, torna-se possível a visualização do sistema de auto pagamento oferecido pelo payback. Em posse dos dados de entrada e saída (cash-flow), é realizável o cálculo da taxa interna de retorno, a qual expressa a remuneração do capital investido. A tabela 5 retrata a situação trabalhada.

Tabela 5: Payback e TIR

\begin{tabular}{|c|c|c|c|c|c|}
\hline Período & Ano & \multicolumn{2}{|c|}{ Cash-flow } & \multicolumn{2}{|c|}{ Payback } \\
\hline 0 & 2017 & $-\mathrm{R} \$$ & $29.247,00$ & $-\mathrm{R} \$$ & $29.247,00$ \\
\hline 1 & 2018 & $\mathrm{R} \$$ & $4.552,50$ & $-\mathrm{R} \$$ & $24.694,50$ \\
\hline 2 & 2019 & $\mathrm{R} \$$ & $4.958,43$ & $-\mathrm{R} \$$ & $19.736,07$ \\
\hline 3 & 2020 & $\mathrm{R} \$$ & $5.400,55$ & $-\mathrm{R} \$$ & $14.335,52$ \\
\hline 4 & 2021 & $\mathrm{R} \$$ & $5.882,10$ & $-\mathrm{R} \$$ & $8.453,43$ \\
\hline 5 & 2022 & $\mathrm{R} \$$ & $6.406,58$ & $-\mathrm{R} \$$ & $2.046,85$ \\
\hline 6 & 2023 & $\mathrm{R} \$$ & $6.977,83$ & $\mathrm{R} \$$ & $4.930,98$ \\
\hline 7 & 2024 & $\mathrm{R} \$$ & $7.600,01$ & $\mathrm{R} \$$ & $4.930,98$ \\
\hline 8 & 2025 & $\mathrm{R} \$$ & $8.277,67$ & $\mathrm{R} \$$ & $20.808,66$ \\
\hline 9 & 2026 & $\mathrm{R} \$$ & $9.015,76$ & $\mathrm{R} \$$ & $29.824,43$ \\
\hline 10 & 2027 & $\mathrm{R} \$$ & $9.819,66$ & $\mathrm{R} \$$ & $39.644,09$ \\
\hline 11 & 2028 & $\mathrm{R} \$$ & $10.695,24$ & $\mathrm{R} \$$ & $50.339,33$ \\
\hline 12 & 2029 & $\mathrm{R} \$$ & $11.648,89$ & $\mathrm{R} \$$ & $61.988,22$ \\
\hline 13 & 2030 & $\mathrm{R} \$$ & $12.687,58$ & $\mathrm{R} \$$ & $74.675,80$ \\
\hline 14 & 2031 & $\mathrm{R} \$$ & $13.818,88$ & $\mathrm{R} \$$ & $88.494,68$ \\
\hline 15 & 2032 & $\mathrm{R} \$$ & $15.051,05$ & $\mathrm{R} \$$ & $103.545,73$ \\
\hline 16 & 2033 & $\mathrm{R} \$$ & $16.393,10$ & $\mathrm{R} \$$ & $119.938,83$ \\
\hline 17 & 2034 & $\mathrm{R} \$$ & $17.854,80$ & $\mathrm{R} \$$ & $137.793,63$ \\
\hline 18 & 2035 & $\mathrm{R} \$$ & $19.446,84$ & $\mathrm{R} \$$ & $157.240,48$ \\
\hline 19 & 2036 & $\mathrm{R} \$$ & $21.180,84$ & $\mathrm{R} \$$ & $157.240,48$ \\
\hline 20 & 2037 & $\mathrm{R} \$$ & $23.069,45$ & $\mathrm{R} \$$ & $201.490,77$ \\
\hline 21 & 2038 & $\mathrm{R} \$$ & $25.126,46$ & $\mathrm{R} \$$ & $226.617,23$ \\
\hline 22 & 2039 & $\mathrm{R} \$$ & $27.366,89$ & $\mathrm{R} \$$ & $253.984,12$ \\
\hline 23 & 2040 & $\mathrm{R} \$$ & $29.807,08$ & $\mathrm{R} \$$ & $283.791,21$ \\
\hline 24 & 2041 & $\mathrm{R} \$$ & $32.464,86$ & $\mathrm{R} \$$ & $316.256,07$ \\
\hline 25 & 2042 & $\mathrm{R} \$$ & $35.359,63$ & $\mathrm{R} \$$ & $351.615,70$ \\
\hline TIR & & & & & \\
\hline
\end{tabular}

Fonte: Autores, (2018). 
Para melhor compreensão e buscando uma forma mais intuitiva de organizar os dados, a figura 3 dispõe de um gráfico para ilustrar, em um intervalo de 25 anos, o tempo que o investimento leva para compensar o recurso empregado.

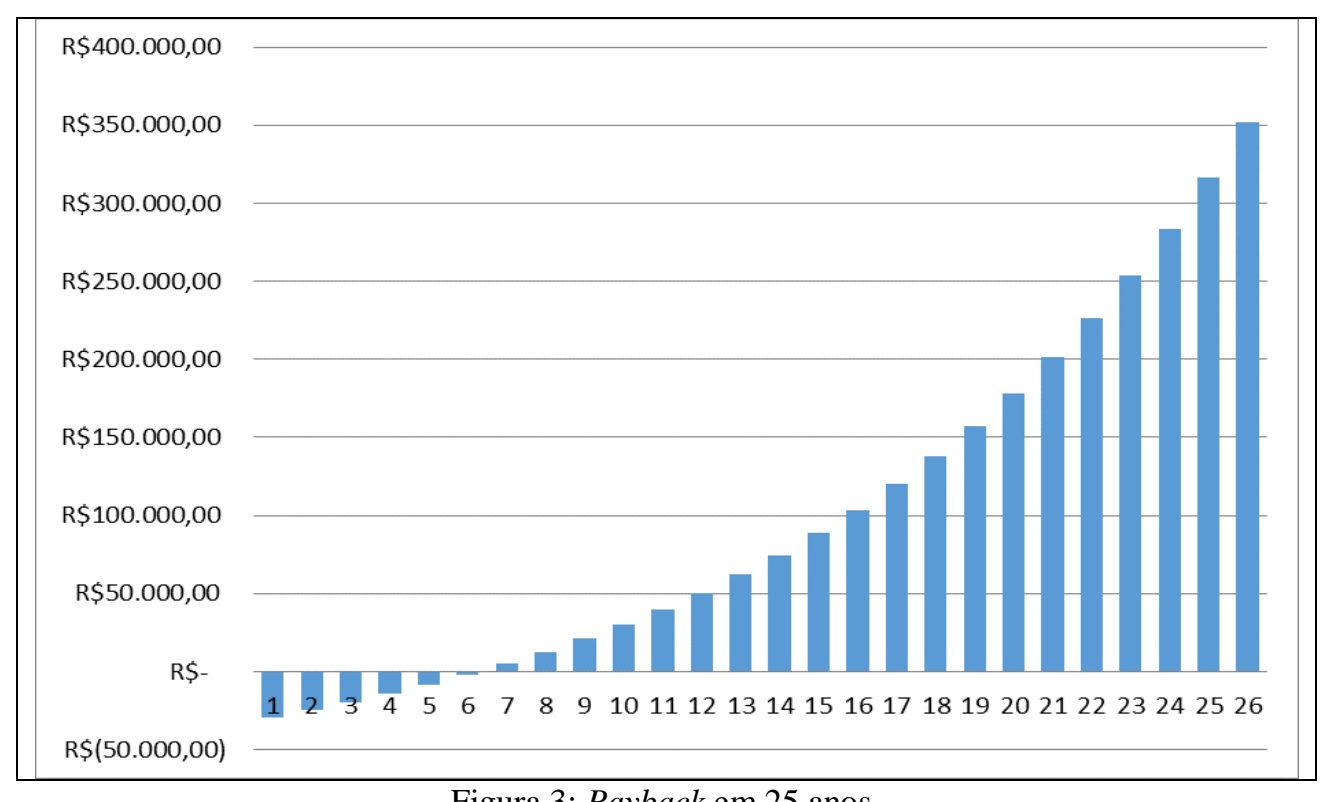

Figura 3: Payback em 25 anos.

Fonte: Autores, (2018).

De modo a dar um caráter diferenciado e prover maior quantidade de indicadores, assim como facilitar o processo decisório, ainda há abertura a mais um recurso avaliativo, o índice de lucratividade. De acordo com [16], o Índice de Lucratividade (IL) é uma medida relativa entre o valor presente dos fluxos de caixa recebidos e o investimento inicial. A equação abaixo retrata a situação.

$$
I L=\frac{V P L}{I_{0}}
$$

Sendo $V P L$ o valor presente líquido e $I_{0}$ o investimento inicial.

O critério de decisão é muito simples. O investimento deve ser aceito se ao menos $I L=1$. Isso significa que a soma dos fluxos de caixa, descontados pela taxa escolhida, será pelo menos igual ao investimento inicial [17].

Aplicando os valores das variáveis à fórmula, obtém-se um IL de 3,71, isto é, para cada R \$ 1,00 investido, o retorno gerado pelo sistema fotovoltaico é de $\mathrm{R} \$ 3,71$. Assim, esse indicador solida os resultados anteriores, no tocante à rentabilidade, enfatizando a viabilidade nessa situação.

Apesar de raramente utilizado, em virtude de sua simplicidade, tal índice é capaz de fornecer dados que podem agregar informações no que tange aos critérios de avaliação do investimento.

Diante dos resultados expostos, pode-se afirmar, com base nos parâmetros adotados, que o investimento possui liquidez, e grande retorno do capital injetado, além de um tempo de payback considerado bastante atrativo, além de utilizar uma fonte de energia considerada infinita. Como citado anteriormente nesta pesquisa, tal atratividade é fruto da desconstrução de barreiras econômicas e sociais, as quais limitavam e restringiam a utilização desse novo modelo de energia. Em virtude disso, se faz necessária uma difusão dessa prática, tendo como foco apresentar os aspectos rentáveis, e assim, incentivar esse modelo de geração.

\section{CONSIDERAÇÕES FINAIS}

O presente estudo obteve êxito em realizar a análise econômico-financeira proposta, empregando os métodos de análise de investimento descritos, no objeto de estudo em destaque, bem como identificar os pontos favoráveis da utilização da energia fotovoltaica. A isenção de ICMS sobre a microgeração no estado de Pernambuco foi o fator primordial para que o tempo de payback se tornasse tão atrativo, visto que essa liberação ocasiona um aumento na economia anual, a qual tem influência direta neste indicador econômico. Em suma, nas condições em que a simulação foi realizada, é notória a viabilidade de investimento. Vários aspectos contribuíram significativamente para a obtenção de resultados de tal magnitude, além da isenção de ICMS, como citado, o aumento médio da tarifa de energia, o qual foi um valor alto em virtude das alterações nos últimos anos, fez elevar as projeções do fluxo de caixa, o que acarretou em uma maior rapidez no tempo de pagamento. Ademais, a taxa mínima de atratividade escolhida nesse caso, foi baixa em comparação à outros estudos presentes na literatura acerca da temática, porém, as razões para escolha foram sólidas. $\mathrm{O}$ valor utilizado foi o rendimento médio da poupança, caso a proposta se mostrasse inviável, seria mais proveitoso aplicar o recurso no banco do que empregá-lo na aquisição de um sistema fotovoltaico. Visto que a designação da TMA é algo que não segue regras, mas sim um critério de decisão, cabe somente ao investidor definir. É válido salientar que, as projeções aqui feitas são baseadas em um consumo de até $584 \mathrm{kWh}$ mensais para o tipo de classificação destacada na seção 4 deste trabalho, sendo necessário outro estudo, caso não se enquadre às especificações utilizadas. Devido à flutuação constante das taxas do mercado, tais projeções se caracterizam como aproximações, podendo não ser os futuros valores reais. Isto pode configurar-se como limitação do presente artigo. 


\section{REFERÊNCIAS}

[1] ANEEL. Resolução Normativa $n^{\circ} 687$, de 24 de Novembro de 2015. 2015. Disponível em: <http://www2.aneel.gov.br/cedoc/ren2015687.pdf>. Acesso em 31 ago 2017.

[2] ANEEL. Procedimentos de Distribuição de Energia Elétrica no Sistema Elétrico Nacional (PRODIST): Módulo 1 Introdução. 2016.2 Disponível em: <http://www.aneel.gov.br/documents/656827/14866914/M\%C 3\%B3dulo1_Revisao_9/1b78da82-6503-4965-abc1a2266eb5f4d7>. Acesso em 18 jul. 2017.

[3] ANEEL. Resolução Normativa no 414, de 9 de Setembro de 2010.2010 .2 Disponível em: <http://www2.aneel.gov.br/cedoc/ren2010414.pdf>. Acesso em 18 jul. 2017.

[4] Portal Solar. Placa Solar Preço. 2015. Disponível em: <http://www.portalsolar.com.br/placa-solar-preco.html>. Acesso em 16 jul. 2017.

[5] Nakabayashi, R.; Microgeração fotovoltaica no Brasil: Viabilidade econômica. 2015. 107 p. Dissertação (Mestrado em Ciências) - Instituto de Energia e Ambiente. Universidade de São Paulo, São Paulo. 2015

[6] Portal Solar. Escolhendo o Painel Fotovoltaico - 10 Coisas Para Saber. 2014. Disponível em: <http://www.portalsolar.com.br/escolhendo-o-painelfotovoltaico--10-coisas-para-saber.htm>. Acesso em 16 jul. 2017.

[7] Santos, F. A.; Souza, C.A.; Dalfior, V. A. O.; ENERGIA SOLAR: um estudo sobre a viabilidade econômica de instalação do sistema fotovoltaico em uma residência em Ipatinga-MG. Em: Simpósio de excelência em Gestão e Tecnologia, XIII, Rio de Janeiro, RJ, 2016. 14 p.

[8] Dassi, J. A.; Zanin, A.; Bagatini, F. M.; Tibola, A.; Barichello, R.; Moura, G. D.D.; Análise da viabilidade econômico-financeira da energia solar fotovoltaica em uma Instituição de Ensino Superior do Sul do Brasil. Em: Congresso Brasileiro de custos, XXII, Foz do Iguaçu, PR, 2015. 16 p.

[9] Kauark, F.; Manhães, F. C.; Medeiros, C. H.; Metodologia da Pesquisa: Um guia prático. Itabuna: Via Litterarum, 2010.

[10] Puccini, E. C.; Matemática Financeira e Análise de Investimentos, Departamento de Ciências da Administração. Universidade Federal de Santa Catarina, Florianópolis. CAPES: UAB, 2011. 204 p.

[11] Bona, A.; Conheça os métodos de análise de investimentos. 2016.2 Disponível em: <https://andrebona.com.br/analise-de-investimentos/>. Acesso em 26 jul. 2017.

[12] Bona, A.; Como saber se o meu investimento está dando certo. 2016. Disponível em: <https://andrebona.com.br/comosaber-se-o-meu-investimento-esta-dando-certo/>. Acesso em 26 jul. 2017.
[13] Warren, C. S.; Reeve, J. M.; Fess, P. E.; Contabilidade Gerencial. São Paulo: Pioneira Thomson Learning, 2001.

[14] Pilão, N. E.; Hummel, P. R. V.; Matemática financeira e engenharia econômica: a teoria e a prática da análise de projetos de investimento. São Paulo: Pioneira Thomson Learning, 2004.

[15] Mesquita, R.; Payback: O que é e como calcular o da sua empresa. Disponível em:〈http://saiadolugar.com.br/payback/〉. Acesso em 26 jul. 2017.

[16] Reis, V. V; Valverde, A. R.; Mendonça, R. R. de; Viabilidade econômica de um projeto de microgeração fotovoltaica residencial no ambiente de compensação de energia elétrica. In: Congresso Virtual Brasileiro de Administração. XII. 2015.

[17] Bordeaux-Rêgo, R.; Paulo, G. P.; Spritzer, I. M. De P. A.; Zotes, L. P.; Viabilidade econômico-financeira de projetos. Rio de Janeiro: Editora FGV, 2006. 